\title{
Seasonal forecast of French Mediterranean heavy precipitating events linked to weather regimes
}

\author{
J.-F. Guérémy ${ }^{1}$, N. Laanaia ${ }^{1}$, and J.-P. Céron ${ }^{2}$ \\ ${ }^{1}$ GAME/CNRM, CNRS/INSU Groupe d'étude de l'atmosphère météorologique (GAME), URA1357, Toulouse, France \\ ${ }^{2}$ DCLIM, Météo-France, Toulouse, France \\ Correspondence to: J.-F. Guérémy (jean-francois.gueremy@meteo.fr)
}

Received: 4 November 2011 - Revised: 2 April 2012 - Accepted: 14 June 2012 - Published: 25 July 2012

\begin{abstract}
Seasonal predictability of local precipitation is rather weak in the mid-latitudes. This is the case when assessing the skill of the seasonal forecast of Heavy Precipitating Event (HPE) extreme occurrence over the French Mediterranean coast during the fall season. Tropics to extratropics teleconnection patterns do appear when averaging analyzed fields over the years characterised by a frequency of HPE occurrence in the upper $17 \%$ of the distribution. A methodology taking weather regime occurrence into account as an intermediate step to forecast HPE extreme occurrence is presented. For the period 1960 to 2001 and four different sets of seasonal forecast, the Economical Value is doubled, compared to the score obtained with the simulated local precipitation data, when using a linear model (Linear Discriminant Analysis in this case) taking simulated $200 \mathrm{hPa}$ velocity potential-stream function regime occurrences as predictors. Interestingly, larger scores are shown for this couple of fields over a large-scale domain including the tropics than for the $500 \mathrm{hPa}$ geopotential height over an Euro-Atlantic domain, despite a tighter link of the latter field to the local precipitation.
\end{abstract}

\section{Introduction}

The primary source of seasonal predictability lies in the tropics where large-scale atmospheric divergent circulation is driven by surface temperatures which often present long-lived anomalies over the oceans. The most prominent anomalous phenomenon is the El Nino Southern Oscillation (ENSO) affecting the Pacific Ocean. Anomalous divergent tropical circulations often trigger anomalous rotational circulations in the mid-latitudes in the form of Rossby wave trains, which in turn affect the climate of these regions. Seasonal forecasts present some skill in the extra-tropics due to this teleconnection process highlighted by the pioneering work of Gill (1980).

Tropical remote impact on the Mediterranean climate has already been described by some studies (Park, 2004; Shaman and Tziperman, 2011). Both studies propose that an eastward Rossby wave train emanating from the Pacific during autumn might influence the Mediterranean climate. Over the Atlantic off the European coast, this barotropic wave train ends with a north-south dipole of vorticity anomaly (positive lobe in the north, just southwest of Ireland, and negative lobe in the south, west of the Straits of Gibraltar, from a Niño minus Niña composite), leading to an increase of westerly moisture advection at $40^{\circ} \mathrm{N}$.

Interestingly, results obtained in the frame of the EU project DEMETER (Palmer et al., 2004) showed some skill in the seasonal forecast of the raw coupled model precipitation field in autumn over the French Mediterranean coast (i.e. correlation larger than 0.2, http://www.ecmwf.int/ research/demeter/index.html).

Instead of considering a mean seasonal anomaly, it could be more fruitful to take into account the seasonal anomaly of weather regime occurrence. The concept and study of weather regimes have been reported by several authors since the 1980s (e.g. Vautard and Legras, 1988; Michelangeli et al., 1995; Cassou et al., 2004; Chabot, 2008). Considering weather regimes could bring two advantages. First, it provides additional information on the predicted distribution beyond only the mean. Second, it offers the possibility to build statistical adaptation in order to forecast local scale phenomena. 
The main goal of the present study is to assess the seasonal predictability of the occurrence of Heavy Precipitating Events (hereafter called HPE) over southeastern France during fall season (September to November of the years 1960 to 2001, as defined in Nuissier et al., 2011). More precisely, the target event is defined as the years for which the HPE occurrence is greater than the mean plus one standard deviation. A direct methodology is performed, taking into account the raw precipitation rates over the considered area from the coupled models used in seasonal forecast. As the region of interest is rather small and as the precipitation field is rather difficult to predict, an indirect methodology is designed in order to take advantage of the better predictability of a larger scale from the dynamics. The model dynamics are considered in terms of large-scale weather regimes which are then statistically linked to HPE over the local region. Thus, the question is whether the scores obtained with the indirect methodology will be larger than those obtained making use of the direct one.

Section 2 describes the datasets used for this work. In Sect. 3, we define the considered weather regimes and we assess their seasonal predictability. Section 4 is devoted to the assessment of HPE occurrence seasonal predictability. Finally, Sect. 5 presents the conclusion and prospects of this work.

\section{Data}

Four sets of coupled seasonal forecast are taken into account ( 9 members per ensemble), using the ECMWF ERA40 reanalysis (Uppala et al., 2005) as a reference. Two of them have been produced in the frame of the EU project ENSEMBLES (Hewitt and Griggs, 2004) - one with the MétéoFrance model $(310 \mathrm{~km}$ grid size, hereafter called MF) and the second with the ECMWF model $(125 \mathrm{~km}$ grid size, hereafter called EC). The other two sets were produced in the frame of the French MEDUP project (which sponsored the present work) using the previous ENSEMBLES MétéoFrance model, with a stretched geometry focused over the Mediterranean sea $(50 \mathrm{~km}$ to $310 \mathrm{~km}$ grid size, hereafter called MED) on one hand, and with a new physics package described in Guérémy (2011) $(155 \mathrm{~km}$ size, hereafter called NPIR) on the other hand. Each member corresponds to a coupled ocean-atmosphere simulation starting at the beginning of August and lasting for at least 4 months in order to consider the fall season, defined by the months 2 to 4 (i.e. September to November). A given member differs from others in terms of a few days lag in the initial conditions, plus stochastic physics in the course of the simulation for the EC set. The four sets cover a 42-yr period (1960 to 2001).

The HPE data are those used in Nuissier et al. (2011) over the same period (i.e. September to November of years 1960 to 2001). Rainfall observations are provided by the MétéoFrance rain gauge network. 435 stations are retained along the French Mediterranean coast including the Pyrenees and Massif Central foothills (Fig. 1b of Nuissier et al., 2011), for which the $99 \%$ quantile is greater than $70 \mathrm{~mm}$ and at least one daily record exceeds $170 \mathrm{~mm}$. By definition, a HPE is occurring during any day, if at least 2 stations (distant from at least $60 \mathrm{~km}$ ) reach their corresponding $99.9 \%$ quantile. Over the 1960-2001 period, 177 HPE occurred, which is a mean of 4.2 per year during the fall season. The standard deviation is of the order of 3. Thus, $7 \mathrm{yr}$ (out of 42) present a HPE occurrence greater than the mean plus one standard deviation.

\section{Seasonal predictability of weather regimes}

\subsection{Large-scale fields considered}

To characterise weather regimes, two dynamical fields were chosen. Firstly, a rather classical field was considered, namely the geopotential height at $500 \mathrm{hPa}$ (hereafter called Z500) over a region covering the northern Atlantic and Europe $\left(25^{\circ}-80^{\circ} \mathrm{N}\right.$ and $90^{\circ} \mathrm{W}-40^{\circ} \mathrm{E}$, using a $2.5^{\circ} \times 2.5^{\circ}$ latitude-longitude grid). Two reasons influenced this choice: first, earlier studies also used this field (e.g. Cassou et al., 2004), and second, it seemed obvious for finding a significant link between the HPE occurrence and such a regional field - this link has already been shown in Nuissier et al. (2011). Secondly, a couple of fields was chosen, including the velocity potential together with the stream function at $200 \mathrm{hPa}$ (hereafter called $\chi \psi 200$ ), over a domain covering both the tropics and the Northern-Hemisphere $\left(15^{\circ} \mathrm{S}-80^{\circ} \mathrm{N}\right.$ and $180^{\circ} \mathrm{W}-180^{\circ} \mathrm{E}$; using a $2.5^{\circ} \times 2.5^{\circ}$ latitude-longitude grid). This couple of scalar fields defines entirely the wind through these gradients (sum of the divergent and rotational wind); it has the advantage of continuously representing the teleconnections between the tropics and mid-latitudes, starting with divergent circulations described by $\chi$ over the tropics and followed by rotational wave trains, associated with the previous divergent circulations, described by $\psi$ over the extra-tropics.

\subsection{Composite analysis}

Prior to the assessment of seasonal predictability of the weather regimes, a study of the teleconnection patterns associated to a frequency of HPE occurrence larger than the mean plus one standard deviation (the last "sextile", the upper $17 \%$ of the distribution) was carried out. These teleconnection patterns were obtained by averaging several fields over the years characterised by a frequency of HPE occurrence being in the upper $17 \%$ of the distribution. Figure 1 shows the composite anomalies of Z500, the large-scale field characterised by the larger a priori link to the HPE (the statistically significant patterns are delimited with black solid lines, from a Student confidence interval). Concerning ERA40, it appears over the Euro-Atlantic domain as an Atlantic low regime (also called Scandinavian blocking) pattern: negative values in the 


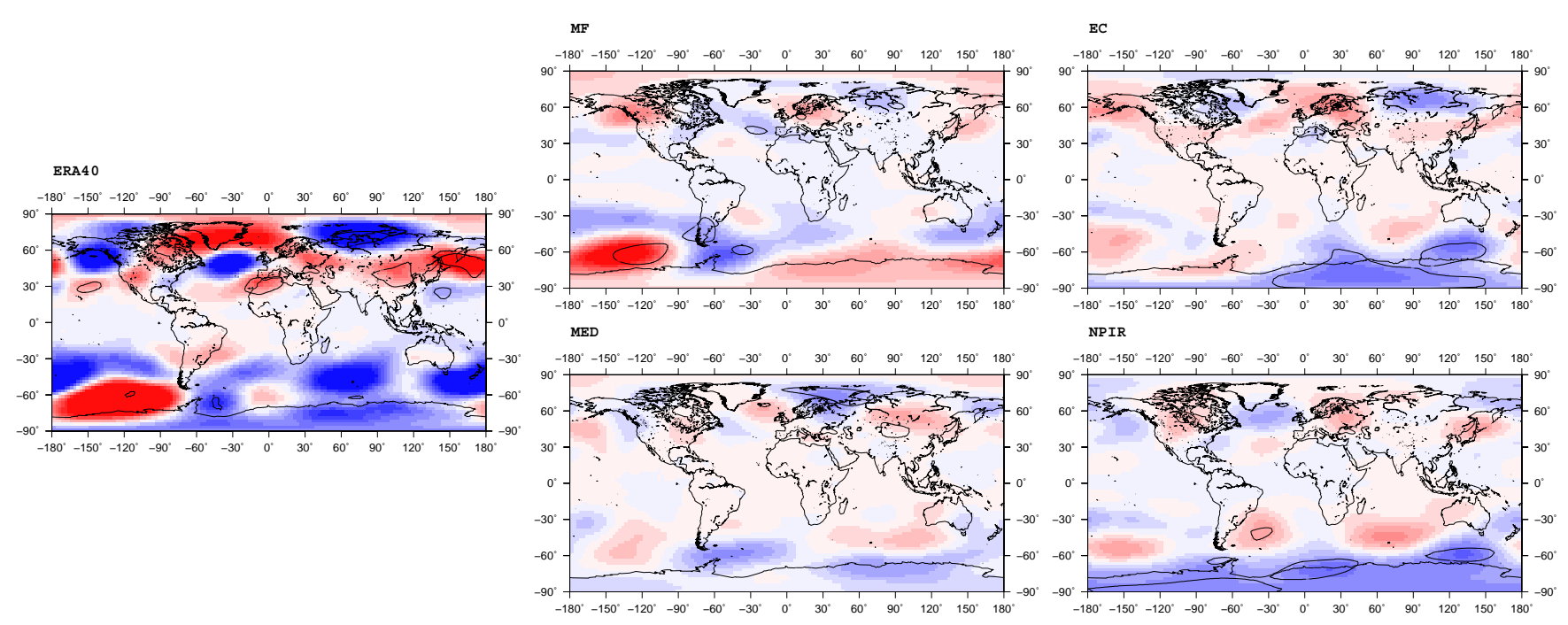

Fig. 1. Z500 anomaly composites (red: +, blue: -) built from averaging Z500 anomalies over the years characterised by a frequency of HPE occurrence being in the upper $17 \%$ of the distribution; from left to right and top to bottom ERA40, MF, EC, MED, and NPIR. Contour interval $2 \mathrm{~m}$. The statistically significant patterns are delimited with black solid lines, from a Student confidence interval.

Atlantic between Iceland and Azores, surrounded by positive values on the western side both in the north and in the south). MF and NPIR provide the best reproduction of this tripole pattern; considering the whole Northern-Hemisphere (notably upstream of the Atlantic, over the Pacific and Northern America), NPIR is the closest to ERA40. This pattern from the Pacific to the Atlantic is very similar to the one described in Shaman and Tziperman (2011) based on a composite (Niño minus Niña) of vorticity anomalies; indeed, our selected $7 \mathrm{yr}$ are rather characterised by positive Sea Surface Temperature (SST) anomalies in the Pacific (see in the following).

In a trial to track the teleconnection patterns from the tropics to the mid-latitudes, the following composite fields were plotted (not shown): starting with the SST, then precipitation (directly influenced by SST in the tropics) followed by $\chi \psi 200$ (ideal couple of fields to track the teleconnections). ERA40 SST anomalies were positive in the centre of the equatorial Pacific and negative over the maritime continent. In relation to SST anomalies, the same kind of precipitation anomaly patterns of the same sign were present both in ERA40 and in the forecasts. Furthermore, $\chi$ anomaly patterns in the tropics were very similar to the ones of precipitation with an opposite sign (negative $\chi$ values for divergent circulation on top of regions of positive precipitation anomalies), with a wave-number one spatial structure (negative over the equatorial central Pacific and positive elsewhere). Finally, $\psi$ anomaly patterns were related to $\chi$ ones with positive (negative) values polar-ward of $\chi$ positive areas in the Northern- (Southern-) Hemisphere, followed by wave trains extending in the mid-latitudes. As expected, the $\psi 200$ pattern in the extra-tropics was very close to that of Z500 (Fig. 1), indicative of a barotropic response. Indeed, over the northwestern hemisphere, ERA40 exhibited a weak Pacific North Atlantic (PNA) pattern elongated to the east into an Atlantic-low-Scandinavian-high pattern, as in Fig. 1 (see Fig. 4). Only NPIR was able to partly reproduce such a spatial structure.

\subsection{Two sets of weather regimes}

Two sets of weather regimes were considered, as indicated above, Z500 over an Euro-Atlantic domain and $\chi \psi 200$ (two fields taken together) over a tropics-Northern-Hemisphere region. At each grid-point a mean (over $42 \mathrm{yr}$ ) linear tendency together with a mean seasonal cycle were subtracted from the raw data, which was then divided by its standard deviation, to apply a Principal Component Analysis (PCA) on daily normalised values. Out of this PCA, only the first tenth of PCs were retained to compress the information while keeping the main total variance. A k-means clustering procedure was applied to the PCs to finally get a set of spatial clusters after projection onto the corresponding Empirical Orthogonal Functions (EOF). Four clusters were retained for Z500, corresponding to the 2 first EOFs (about $22 \%$ of the total variance) with plus and minus signs. Figure 2 shows ERA40's four clusters and their corresponding frequency of occurrence. The first and third regimes (clusters) represent the North Atlantic Oscillation (NAO) circulation types; the second and fourth regimes represent meridian types of circulation (versus NAO zonal types), Atlantic Low (AL, also known as Scandinavian blocking) and Atlantic Ridge (AR), respectively. Not surprisingly, among the total amount of 177 observed HPE in the period 1960 to 2001, $57 \%$ occur during AL days (days belonging to AL regime), $19 \%$ during NAO-, and $12 \%$ during both NAO+ and AR. The $\mathrm{Z} 500$ regimes 

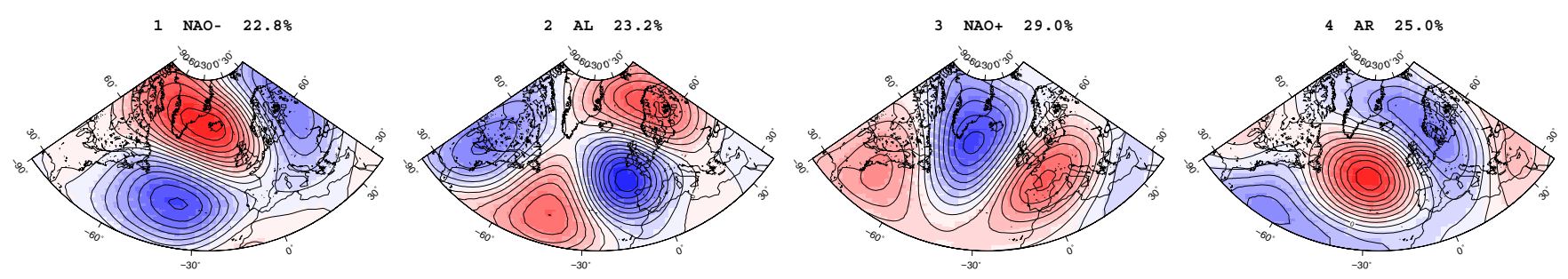

Fig. 2. ERA40 Z500 clusters (NAO-, Atlantic Low, NAO+, Atlantic Ridge) and their corresponding frequency of occurrence. Contour interval 0.1 (normalised variable).

defined with each forecast ensemble (considering all 9 member daily values) resemble more or less ERA40 ones (discrepancies exist in spatial location, intensity and occurrence frequency).

Six clusters were retained for $\chi \psi 200$, corresponding to the 3 first EOFs (about $45 \%$ of the total variance) with plus and minus signs. Figure 3 shows ERA40 six clusters for both fields $\psi$ and $\chi$ coming from the combined $\chi \psi 200$ clustering procedure. $\chi$ regimes appeared as a propagative phenomenon from west to east $(1,2,3,4,6,5)$ with a wave-number one; this was corroborated by a spectral analysis of the 3 corresponding PCs, which presented a maximum of variance between 20 to 40 days (not shown). This regime decomposition represents the propagation of the so-called eastward intraseasonal oscillation (also known as the Madden-Julian Oscillation, MJO). $\psi$ regime patterns were characterised by positive values in the Northern Hemisphere (anticyclonic circulations) to the west of (or collocated with) negative values of $\chi$ (divergent circulations), as expected. Some $\psi$ regimes exhibited wave-train patterns in the extra-tropics, such as clusters 1 and 6 for example. Indeed, for these two clusters, the mid-latitude propagation was facilitated by the presence of westerly winds at $200 \mathrm{hPa}$ in the considered region (eastern Pacific and Atlantic), as stated in Lau and Lim (1984). The $\psi 200$ composite of ERA40 is shown in Fig. 4. The pattern is very similar to its counterpart for Z500 (Fig. 1), with more details in the tropics as expected. It is also the closest to cluster 6 and to cluster 1 . This is corroborated by the fact that among the total amount of 177 observed HPE in the period 1960 to 2001, $24 \%$ occured during cluster 1 days (days belonging to regime 1 ) and $21 \%$ occured during cluster 6 days, with less than $16 \%$ for each of the other regimes.

\subsection{Predictability of weather regimes}

In order to assess the predictability of these two sets of regimes (Z500 and $\chi \psi 200$ ), two different scores were computed over the period 1960-2001. First, correlations of frequency of occurrence were calculated for each regime, and second, the probabilistic Relative Operating Characteristic (ROC) score was computed. The ROC score provides the hit rates versus the false alarm rates for a particular event (here, the frequency of occurrence being in the last sextile), a
Table 1. Correlation of frequency of occurrence.

\begin{tabular}{lrrrr}
\hline Z500 & MF & EC & MED & NPIR \\
\hline Cluster 1 & 0.05 & -0.11 & 0.03 & 0.01 \\
Cluster 2 & 0.06 & 0.01 & -0.14 & 0.05 \\
Cluster 3 & 0.03 & -0.01 & 0.18 & 0.20 \\
Cluster 4 & 0.20 & 0.02 & 0.15 & 0.37 \\
\hline$\chi \psi 200$ & MF & EC & MED & NPIR \\
\hline Cluster 1 & 0.45 & 0.38 & 0.40 & 0.41 \\
Cluster 2 & 0.17 & 0.30 & 0.19 & 0.28 \\
Cluster 3 & 0.12 & -0.11 & -0.01 & -0.11 \\
Cluster 4 & -0.09 & 0.39 & 0.01 & 0.05 \\
Cluster 5 & 0.19 & 0.13 & -0.05 & -0.08 \\
Cluster 6 & 0.49 & 0.23 & 0.42 & 0.39 \\
\hline
\end{tabular}

function of a probability threshold defined by the number of members (among 9) of the ensemble forecasting the event. It is noteworthy to remark that confidence intervals were computed for the ROC score (the area under the ROC curve) using a bootstrap procedure.

Table 1 presents the correlation of the frequency of occurrence for Z500 and $\chi \psi 200$. Each day of each member of the 4 sets of seasonal forecast is associated to an ERA40 regime according to the minimum distance (the one used in the clustering procedure) to any of the 4 (6) considered clusters. Over the considered sample of $42 \mathrm{yr}$, correlations below 0.15 were not really significant. Concerning Z500, it is clear that the predictability of cluster 2 (in italic, the one having the largest relation to HPE) is very poor. The only cluster presenting some predictability is 4 , i.e. the Atlantic Ridge; however, this regime is unfavourable to HPE. Interestingly, where $\chi \psi 200$ is concerned, the most predictable clusters are the ones having the largest relation to HPE (i.e. cluster 1 and cluster 6 , in italic). Furthermore, the correlations reach the order of 0.4 , which is a good score in seasonal forecast. Not surprisingly, this score is larger than that obtained with Z500 because of both the larger scale and the a priori tighter link to the predictability source of this couple of variables. Nevertheless, the link between $\chi \psi 200$ and the HPE was coarser than the one obtained with Z500, as stated above. In Sect. 4, the predictability of HPE occurrence using regime occurrence as an 

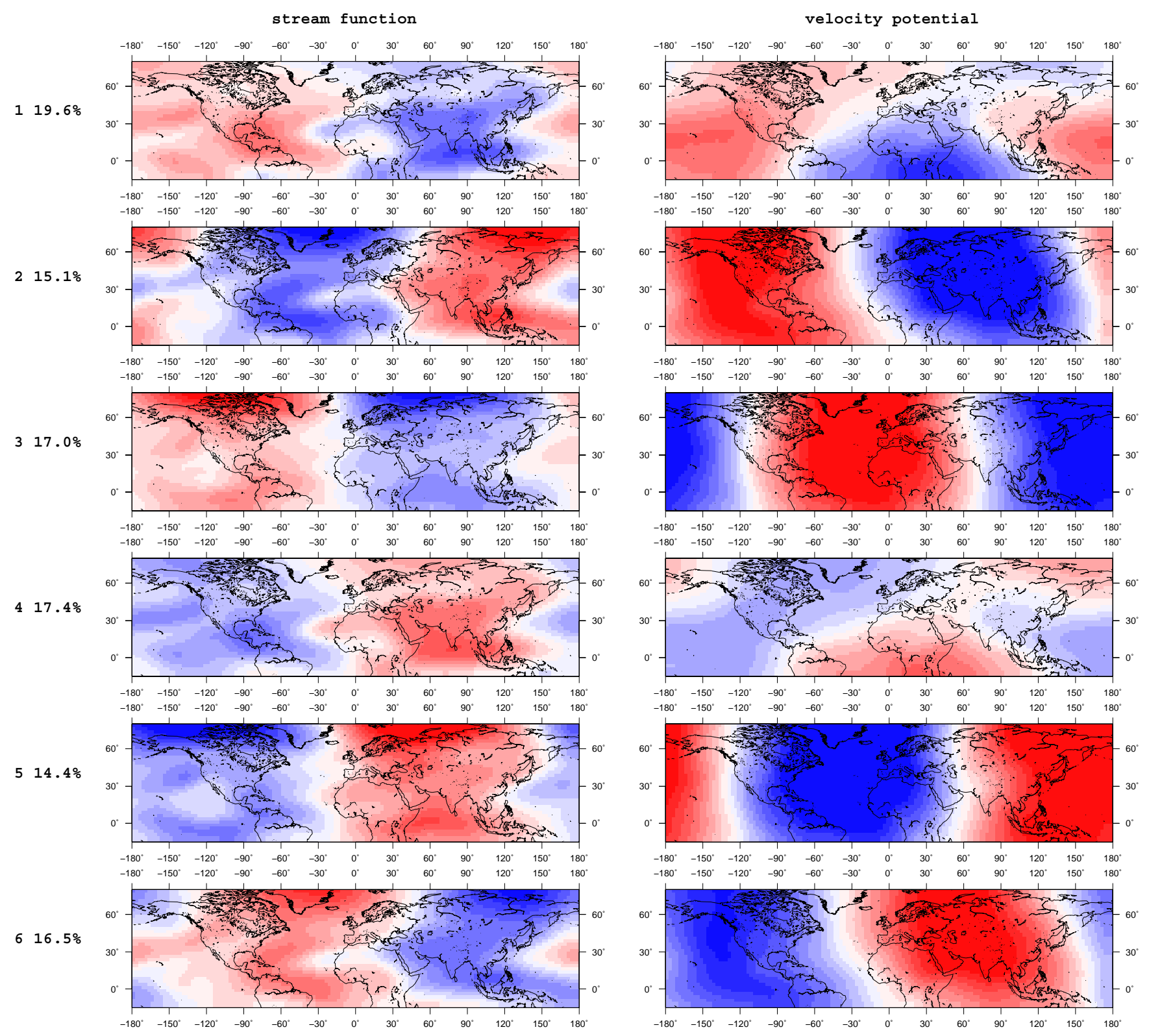

Fig. 3. ERA40 $\chi \psi 200$ clusters and their corresponding frequency of occurrence; stream function on the left and velocity potential on the right. Contour interval 0.1 (normalised variable).

intermediate step will be detailed so as to determine which of the 2 sets of variables provides the best score.

In addition to the correlation scores, ROC scores are shown in Fig. 5 for Z500 and in Fig. 6 for $\chi \psi 200$. Using the climatology, instead of the forecast, would give a curve aligned with the diagonal; therefore, only the ROC curves above the diagonal are indicative of skill. Concerning Z500, the present scores for the event "frequency of occurrence being in the last sextile" are rather poor, except for MED and to a lesser extent for NPIR, as for the correlation scores. The difference between MF and MED is due to the better resolution (the only difference between the two sets of forecasts) of the latter in the region of interest. As with Z500, the best ROC scores were obtained for the $\chi \psi 200$ clusters providing the best correlation scores (i.e. cluster 1 and cluster 6). As is the case for the correlation, the best $\chi \psi 200$ ROC scores are larger than those of Z500. It is worth noting that the best ROC score is obtained by NPIR for the cluster 6 (the one bringing the largest teleconnection signal), even if MF is better in terms of correlation for that particular cluster. 


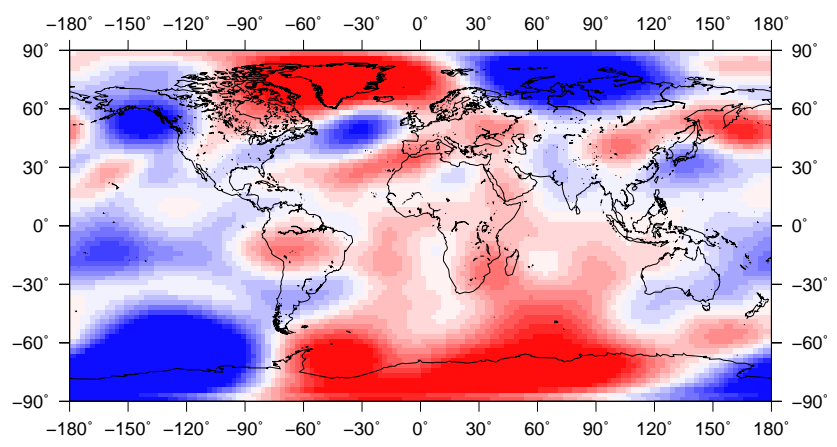

Fig. 4. ERA40 $\psi 200$ anomaly composite built from averaging భ200 anomalies over the years characterised by a frequency of HPE occurrence being in the upper $17 \%$ of the distribution. Contour interval $0.0510^{7} \mathrm{~m}^{2} \mathrm{~s}^{-1}$.
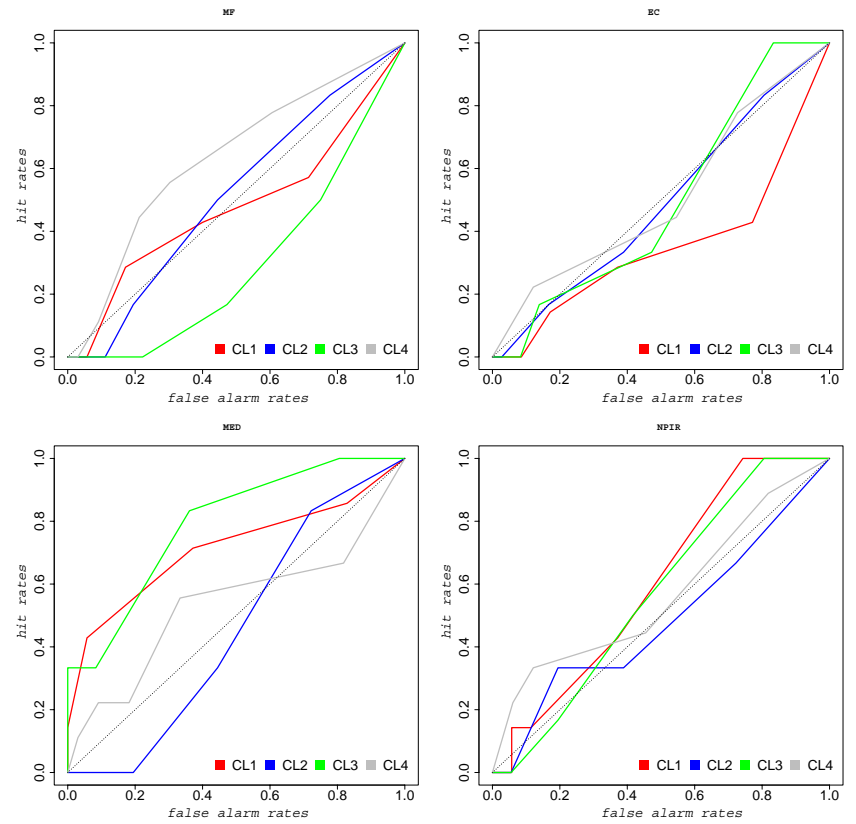

Fig. 5. Z500 ROC curves (false alarm rates in abscissa, hit rates in ordinate) for each cluster, from each forecast set.

\section{Seasonal predictability of HPE}

The aim of this section is to report on the seasonal predictability of HPE occurrence being in the last sextile. In order to define the model predicted HPE, two methods were considered. First, a direct estimation based on the raw precipitation rates over the considered area from the coupled model used in seasonal forecast. Second, two indirect procedures were assessed: firstly, the use of a linear statistical model to link the regime occurrence to the HPE occurrence, and secondly, the use of a non-linear model based on analogue years built with the regime occurrence. The "predictability of the frequency of HPE occurrence being in the last sextile" (our event of interest) was quantified with the help of both the
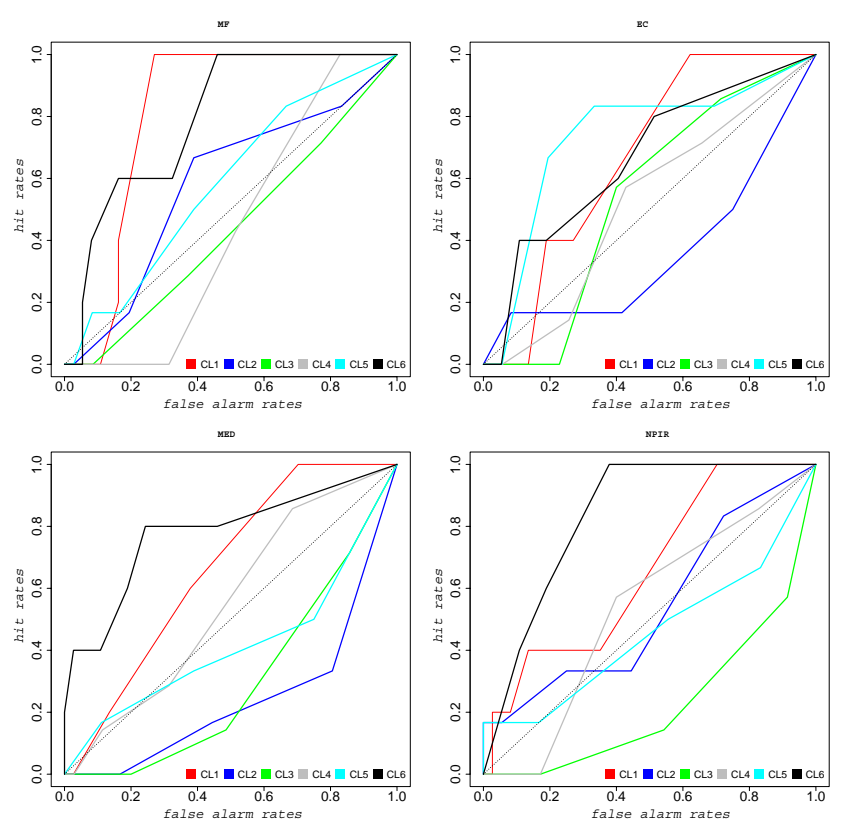

Fig. 6. $\chi \psi 200$ ROC curves (false alarm rates in abscissa, hit rates in ordinate) for each cluster, from each forecast set.
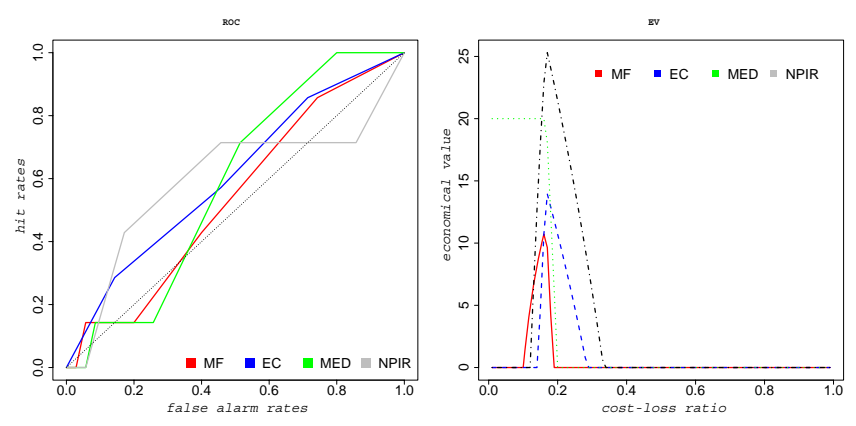

Fig. 7. HPE ROC curves (left; false alarm rates in abscissa, hit rates in ordinate) and Economical Value curves (right; cost-loss ratio in abscissa, economical values in ordinate) for the direct methodology, from each forecast set.

probabilistic Relative Operating Characteristic (ROC) score and the Economical Value score (EV, as in Guérémy et al., 2005). It is noteworthy to remark that confidence intervals were computed for the ROC score (the area under the ROC curve, equal to 0.5 in the case of climatological forecast) using a bootstrap procedure. This confidence interval is particularly relevant to this work in so far as the same database is used to build statistical models and to assess the results. EV provides a measure of the relative gain earned by an end-user taking the seasonal forecast into account, versus climatological and perfect forecasts (scores equal to 0 and 100, respectively). 

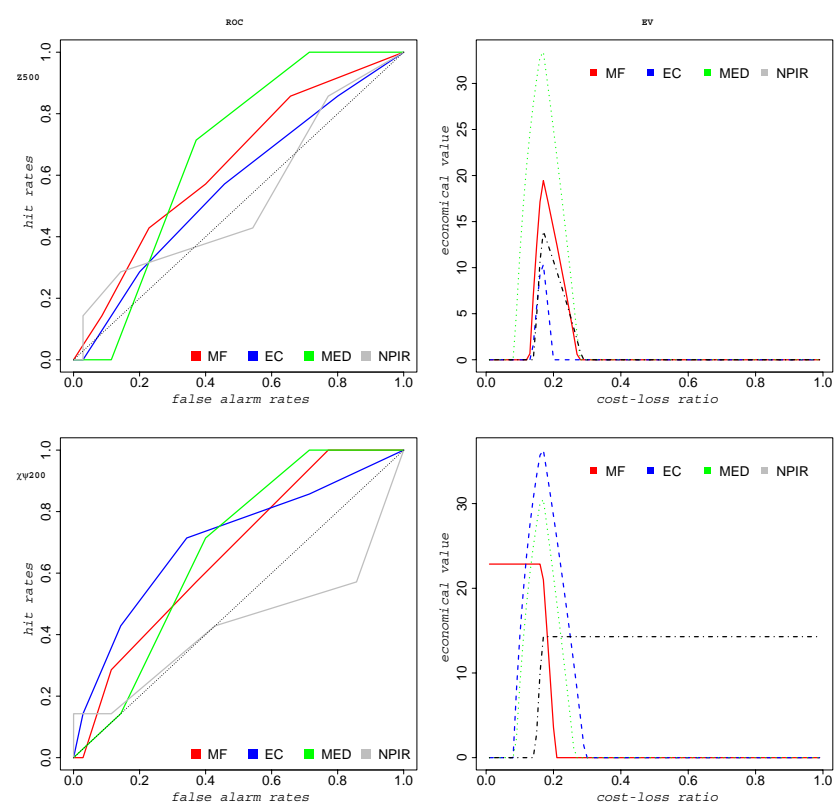

Fig. 8. HPE ROC curves (left; false alarm rates in abscissa, hit rates in ordinate) and Economical Value curves (right, cost-loss ratio in abscissa, economical values in ordinate) for the MOS LRM methodology; Z500 (top), $\chi \psi 200$ (bottom), from each forecast set.
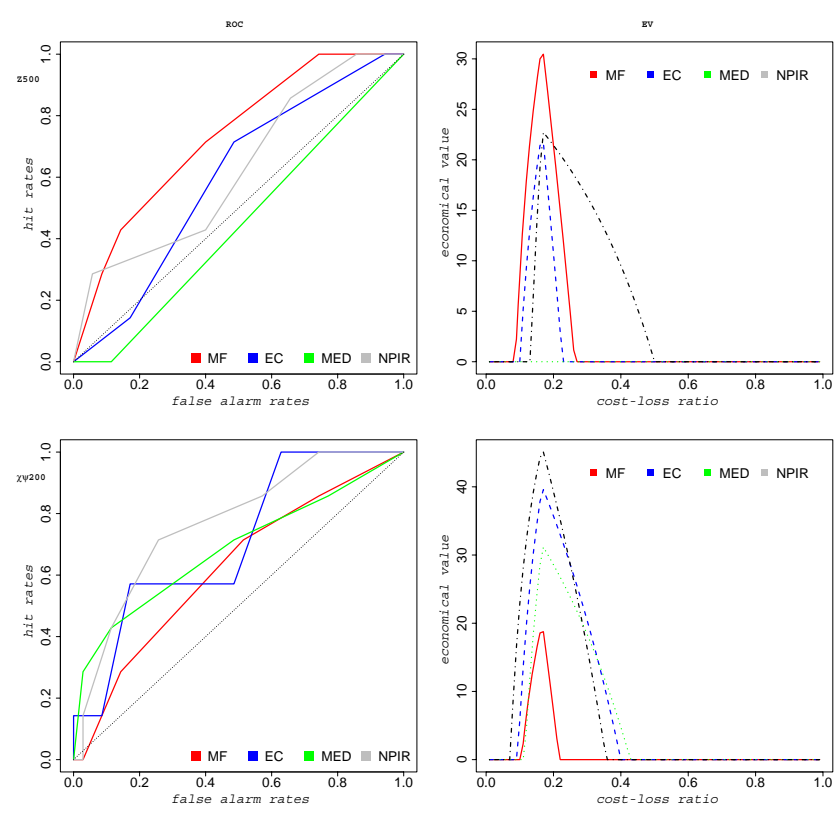

Fig. 9. HPE ROC curves (left; false alarm rates in abscissa, hit rates in ordinate) and EV Economical Value curves (right; cost-loss ratio in abscissa, economical values in ordinate) for the MOS LDA methodology; Z500 (top), $\chi \psi 200$ (bottom), from each forecast set.
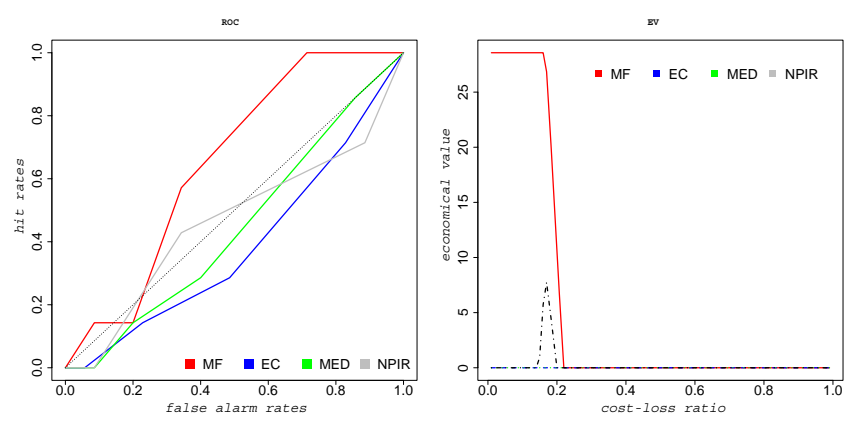

Fig. 10. HPE ROC curves (left; false alarm rates in abscissa, hit rates in ordinate) and Economical Value curves (right; cost-loss ratio in abscissa, economical values in ordinate) for the analogue methodology, from each forecast set.

\subsection{Direct methodology}

For the direct methodology, HPE were defined in the following manner in the forecast simulations: for at least one of the points from the $310-\mathrm{km}$ grid belonging to our region of interest, the daily rain rate had to be larger than the percentile value providing roughly the observed number of HPE (177 over the 42 considered years); for the 4 sets of seasonal forecast, this percentile was of the order of $97 \%$ compared to the value of $99.9 \%$ in the observations. Figure 7 shows the ROC curves of our event of interest (frequency of HPE occurrence being in the last sextile) and the EV curves for the 4 sets of seasonal forecast. The EV is computed as a function of the end-user cost-loss ratio. The cost $\mathrm{C}$ is the amount of money spent in the presence of the event (mitigation purpose), while the loss $\mathrm{L}$ is the amount of money lost in case of non-detection of the event in the forecast. EV is always reaching its maximum value when the ratio $\mathrm{C} / \mathrm{L}$ is equal to the chosen distribution threshold (sextile in our case, i.e. $17 \%$ ). The best score was obtained by NPIR, followed by MED (having the most refined horizontal resolution among the 4 sets). EV scores of the order of $20 \%$ were reached at best, which are rather good scores in seasonal forecasting.

\subsection{Linear indirect methodologies}

The first indirect methodology applied was the linear statistical downscaling. The statistical model was built to get a linear relationship between the frequency of regime occurrence and the frequency of HPE occurrence over the fall season. Two types of statistical models were tested both in Perfect Prog (PP) and Model Output Statistics (MOS) strategies. The first type was the Linear Regression Model (LRM), providing a linear equation between the frequency of regime occurrence and the frequency of HPE occurrence. The second type was the Linear Discriminant Analysis (LDA), providing a linear relation between the frequency of regime occurrence and categories of HPE occurrence. The chosen categories included, obviously, the last sextile (our target in this study) 
Table 2. Area under the ROC curve with a $95 \%$ confidence.

\begin{tabular}{lllll}
\hline & MF & EC & MED & NPIR \\
\hline ROC (area) & 0.62 & 0.71 & 0.68 & 0.77 \\
$95 \%$ Bootstrap & $(0.37,0.83)$ & $(0.48,0.90)$ & $(0.41,0.92)$ & $(0.56,0.94)$ \\
\hline
\end{tabular}

in addition to the first sextile and the category in-between. Both LRM and LDA were tested because the former, being a priori simpler, provided a continuous relationship between predictand and predictors, while the latter seemed a priori more suitable for the present study in so far as it allows for direct consideration of categories of the distribution. The PP strategy consisted of building the statistical model from a reference dataset (here ERA40), while the MOS strategy took the actual forecast dataset into account. Using the LRM, the correlation (over the considered $42 \mathrm{yr}$ ) in terms of frequency of HPE occurrence between ERA40 and local observations reached 0.56 with Z500 and 0.44 with $\chi \psi 200$. As mentioned earlier in the previous section on the predictability of weather regimes, this is a confirmation that the link between Z500 and HPE is larger than the one using $\chi \psi 200$. Concerning the 4 sets of forecasts with the PP strategy, the correlation decreased, reaching 0.18 for MED, 0.11 for NPIR, and 0.09 for both MF and EC when $\chi \psi 200$ was taken into account and equal to zero when Z500 was taken into account. These rather bad results were a consequence of the relatively poor scores of the regime predictability (see the previous section), particularly for Z500. Switching to the MOS strategy, the correlations were much better while being generally less than those obtained with ERA40, reaching up to 0.53 for MF using Z500 and 0.57 for EC using $\chi \psi 200$. Figure 8 shows the ROC curves of our event of interest (frequency of HPE occurrence being in the last sextile) and the EV curves for the 4 sets of seasonal forecast using the MOS strategy. Reasonable scores were obtained with EV of larger than $10 \%$ using the Z500 regime, reminding that the predictability of these regimes is poor (see previous section). When using the PP strategy (not shown), the scores were very poor, consistent with what was found with the correlations. The scores were larger using $\chi \psi 200$, with EV on the order of $25 \%$. These results emphasize the real benefit of the MOS strategy (compared to PP), even if it implies the definition of a linear model per dynamical model, instead of only one linear model defined by ERA40 in the case of PP. Furthermore, they demonstrate the greater potential of $\chi \psi 200$ regimes versus Z500 regimes to predict our event of interest, even if the link between Z500 and HPE is larger than the one using $\chi \psi 200$. The ROC and EV curves of the frequency of HPE occurrence being in the last sextile for the 4 sets of seasonal forecast using LDA together with the MOS strategy are presented in Fig. 9. As was the case with LRM, the use of PP strategy provided very poor scores, with the ROC corresponding to the one for climatology. The results obtained by LDA were sig- nificantly better than those of LRM. Table 2 shows the area under the ROC curve with a $95 \%$ confidence interval provided by a bootstrap procedure. The minimum value of the interval is close to 0.5 (value obtained using the climatology), and NPIR exhibited an even larger value than this climatological threshold. Using $\chi \psi 200$ regimes, the EV reached $40 \%$ (for NPIR and EC) instead of a maximum of $30 \%$ with LRM. The same two remarks mentioned for LRM also stand in this case: there is a significant benefit of the MOS (versus PP) strategy, and mainly there is a greater potential for $\chi \psi 200$ (versus Z500) regimes to predict our event of interest.

\subsection{Analogue indirect methodology}

The second indirect methodology applied was a non-linear model based on analogue years built with the regime occurrence, following a methodology published in Clark and Déqué (2003). Here, the analogue years were selected by picking up the 5 closest years per member in the considered forecast dataset, getting at final 45 analogue years $(9 \mathrm{mem}-$ bers times $5 \mathrm{yr}$ ). Some years could possibly appear several times. The total number of years had been chosen to be on the same order of the total number of different available years. The distance was defined as an euclidean distance based on the frequency of $\chi \psi 200$ regime occurrence (6 clusters). This couple of variables was chosen because the goal was really to search for analogue years in terms of a global atmospheric response. At the end, the forecast of the frequency of HPE occurrence was the one obtained by averaging the actual observed frequency over the 45 selected years. Figure 10 shows the ROC curves and the EV curves of our event of interest for the 4 sets of seasonal forecast. The results are clearly worse than those obtained with a linear model. For such an analogue method, it might be necessary to have a larger number of years to be able to really discriminate different types of atmospheric response.

\section{Conclusion and prospects}

The results presented here indicate that there is a real potential for the seasonal prediction of HPE extreme occurrence over the French Mediterranean coast during the fall season. These results corroborate the findings of earlier studies (e.g. Park, 2004; Shaman and Tziperman, 2011) showing an atmospheric link between Pacific SST anomalies and precipitation over the Mediterranean Sea. The present work also shows the benefit of the use of large-scale weather regimes to 
improve the skill of the seasonal forecast of such a local field when compared to the direct method of taking the local raw field itself into account. A doubling of the Economical Value is obtained when using the indirect method. This is an extension of previous works reporting on some significant skill in the seasonal forecast of weather regimes (e.g. Chabot, 2008).

Interestingly, the best results in terms of forecasting the frequency of HPE occurrence being in the last sextile were obtained using a Linear Discriminant Analysis from $\chi \psi 200$ regimes with a Model Output Statistics strategy. The Perfect Prog strategy (ERA40 as reference) was less skilful, due to the fact that the predictability of regime occurrence was rather weak, remembering that for each simulated day the assignment to a cluster is defined using the ERA40 regime set. One of the main interesting results of this study was the larger scores obtained in fine using $\chi \psi 200$ regimes instead of that of Z500. Indeed, the link between the Z500 regime and the HPE was stronger, but at the same time the predictability of the former regime was worse than that of the latter. Finally, it appears that the Linear Discriminant Analysis provides better results than the Linear Regression Model. This might be due to the fact that the linear relationship based on categories is more robust than the continuous one. The Météo-France model using the new physics package performed the best when applying this above mentioned indirect methodology. This might be the consequence of best overall tropical atmospheric response obtained with this model, considering the best ROC score obtained for $\chi \psi 200$ cluster 6 (the one bringing the largest teleconnection signal, see Fig. 6).

As prospects for this work, we would like to extend this procedure of using large-scale circulation regime occurrence to predict other local fields (over domains having a few hundred kilometre scale). The stream function at $200 \mathrm{hPa}$ in a large tropical domain (i.e. $40^{\circ} \mathrm{S}$ to $40^{\circ} \mathrm{N}$ ) appears to be a good candidate as the large-scale variable to be used in defining the circulation regimes. It might be simpler and sufficient to only keep the stream function instead of the couple $\chi \psi 200$ taken into account in the present study. Moreover, such a large tropical domain, excluding mid-latitude regions (contrary to what has been done here), should provide even better scores for the stream function regime occurrence itself, as reported in Palmer et al. (2004) for example, and hopefully for the predictand at final.

Acknowledgements. This study has been sponsored by the "Forecast and projection in climate scenario of Mediterranean intense events: Uncertainties and Propagation on environment" (MEDUP) project of the program VMC (Vulnérabilité: Milieux et Climat) from the ANR (Agence Nationale pour la Recherche). The authors thank two anonymous reviewers for useful comments and suggestions on an earlier version of the paper.

Edited by: M.-C. Llasat

Reviewed by: two anonymous referees

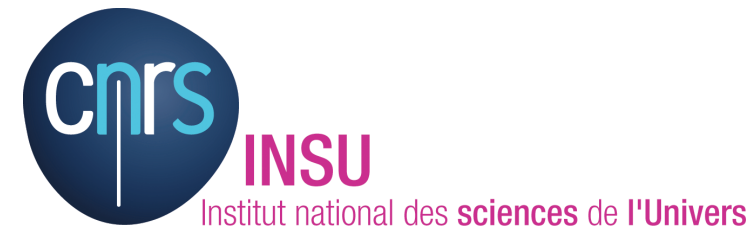

The publication of this article is financed by CNRS-INSU.

\section{References}

Cassou, C., Terray, L., Hurrell, J. W., and Deser, C.: North Atlantic winter climate regimes: Spatial asymmetry, stationarity with time and oceanic forcing, J. Climate, 17, 1055-1068, 2004.

Chabot, E.: Prévisibilité des régimes de temps à échelle saisonnière, Rapport de stage de fin d'études de l'ENM, Toulouse, France, 2008.

Clark, R. T. and Déqué, M.: Conditional probability seasonal predictions of precipitation, Q. J. Roy. Meteorol. Soc., 129, 1-15, 2003.

Gill, A. E.: Some simple solutions for heat-induced tropical circulation. Q. J. Roy. Meteorol. Soc., 106, 447-462, 1980.

Guérémy, J.-F., Déqué, M., Braun, A., and Piedelievre, J.-P.: Actual and potential skill of seasonal predictions using the CNRM contribution to DEMETER: coupled versus uncoupled model, Tellus A, 57, 308-319, 2005.

Guérémy, J.-F.: A continuous buoyancy based convection scheme: one- and three-dimensional validation, Tellus A, 63, 687-706, 2011.

Hewitt, C. D. and Griggs, D. J.: Ensembles-based Predictions of Climate Changes and their Impacts, Eos, 85, 566 pp., 2004.

Lau, K.-M. and Lim, H.: On the Dynamics of Equatorial Forcing of Climate Teleconnections, J. Atmos. Sci., 41, 161-176, 1984.

Michelangeli, P.-A., Vautard, R., and Legras, B.: Weather regimes: Recurrence and quasi-stationarity, J. Atmos. Sci., 52, 12371256, 1995.

Nuissier, O., Joly, B., Joly, A., Ducrocq, V., and Arbogast, P.: A statistical downscaling to identify the large-scale circulation patterns associated with heavy precipitation events over southern France, Q. J. Roy. Meteorol. Soc., 137, 1812-1827, 2011.

Palmer, T. N., Alessandri, A. , Andersen, U., Cantelaube, P, Davey, M., Délécluse, P. Déqué, M,.Díez, E., Doblas-Reyes, F. J., Feddersen, H., Graham, R., Gualdi, S., Guérémy, J.-F., Hagedorn, R.,Hoshen, M., Keenlyside, N., Latif, M., Lazar, A. , Maisonnave, E., Marletto, V., Morse, A. P., Orfila, B., Rogel, P., Terres, J.-M., and Thomson, M. C.: Development of a European MultiModel Ensemble System for Seasonal to Inter-Annual Prediction (DEMETER), Bull. Am. Meteorol. Soc., 85, 853-872, 2004.

Park, S.: Remote ENSO influence on Mediterranean sky conditions during late summer and autumn: Evidence for a slowly evolving atmospheric bridge, Q. J. Roy. Meteorol. Soc., 130, 2409-2422, 2004.

Shaman, J. and Tziperman E.: An Atmospheric Teleconnection Linking ENSO and Southwestern European Precipitation, J. Climate, 24, 124-139, 2011.

Uppala, S. M., KÅllberg, P. W., Simmons, A. J., Andrae, U., Bechtold, V. D. C., Fiorino, M., Gibson, J. K., Haseler, J., Hernandez, A., Kelly, G. A., Li, X., Onogi, K., Saarinen, S., Sokka, N., Allan, R. P., Andersson, E., Arpe, K., Balmaseda, M. A., Beljaars, 
A. C. M., Berg, L. V. D., Bidlot, J., Bormann, N., Caires, S., Chevallier, F., Dethof, A., Dragosavac, M., Fisher, M., Fuentes, M., Hagemann, S., Hólm, E., Hoskins, B. J., Isaksen, L., Janssen, P. A. E. M., Jenne, R., Mcnally, A. P., Mahfouf, J.-F., Morcrette, J.-J., Rayner, N. A., Saunders, R. W., Simon, P., Sterl, A., Trenberth, K. E., Untch, A., Vasiljevic, D., Viterbo, P., and Woollen, J.: The ERA-40 re-analysis, Q. J. R. Meteorol. Soc., 131, 29613012, 2005.
Vautard, R. and Legras, B.: On the source of midlatitude lowfrequency variability. Part II: Nonlinear equilibration of weather regimes, J. Atmos. Sci., 45, 2845-2867, 1988. 